\title{
Objetos de Aprendizagem com Design Thinking
}

\author{
Ivam Galvão Filho"; \\ Vânia Ribas Ulbricht²;
}

resumo:

São inúmeros os desafios enfrentados por educadores na busca por avanços na área da educação e diversas são as ferramentas tecnológicas que podem ser utilizadas para cumprir esse objetivo. Esta situação nos leva a analisar e discutir a questão do ensino-aprendizagem na educação básica, suportada pela tecnologia da comunicação e informação, mais especificamente relacionado ao uso de objetos de aprendizagem (OA). Esse estudo tem, portanto, como objetivo principal, propor o uso do Design Thinking no desenvolvimento de objetos de aprendizagem, com o intuito de realizar melhorias em processos produtivos de OA em termos de Design.

Para alcançar este objetivo, buscou-se realizar uma pesquisa bibliográfica através de uma revisão em bases de dados como a SciElo, Scopus, Web of Science e IEEE Xplore. Todos os trabalhos consultados nas bases de dados foram organizados em planilhas para análise e seleção, junto com outros textos considerados relevantes. Este trabalho resultou na seleção de 80 textos, sendo 71 artigos oriundos da revisão bibliográfica e 9 trabalhos entre artigos e livros que foram integrados ao estudo por serem considerados relevantes para maior compreensão do assunto. A principal conclusão do estudo foi a de que o Design Thinking pode ser utilizado no processo de desenvolvimento de $O A$, de modo a colocar o aluno no centro do processo de desenvolvimento, levando em consideração suas reais necessidades educacionais específicas e fazendo uso das ferramentas disponibilizadas pelo Design Thinking. palavras-chave:

Objetos de Aprendizagem; Educação; Design Thinking

Espaço reservado para organização do congresso.

\footnotetext{
${ }^{1}$ http://lattes.cnpq.br/3032033173877735

${ }^{2}$ http://lattes.cnpq.br/0196218903676581
} 


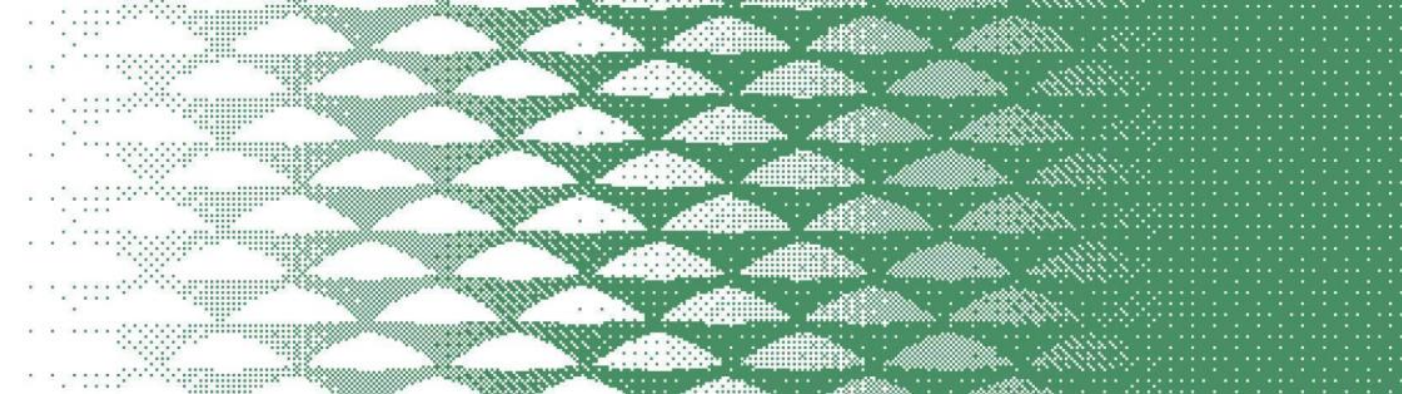

manutenção do padrão de metadados LOM para objetos de aprendizagem —, além das bases de dados da Web of Science (WoS), através da plataforma OUCI e Scopus - duas das mais importantes plataformas virtuais de periódicos científicos no mundo. O período considerado na consulta às bases foi de 2015 a 2020, pois devido a constante evolução tecnológica, um período muito extenso poderia incorrer em levantamento de conceitos em desuso, tanto em relação a tecnologia e design de protótipos, como também com relação a processos de prototipação. Foram considerados na pesquisa somente artigos de acesso público nos idiomas português, inglês e espanhol, e que tratam do tema da pesquisa. Após a consulta, foi realizada uma análise exploratória de dados das publicações, além de uma análise nos títulos, resumos e palavras-chave para verificar a compatibilidade com o tema. Textos repetidos ou sem relação com o tema foram descartados. Outros trabalhos relacionados ao tema foram adicionados à pesquisa de forma empírica.

A seguir, tem-se a apresentação da metodologia utilizada na pesquisa e, em seguida, será apresentada a fundamentação teórica com maiores detalhes sobre a revisão integrativa realizada e uma amostra dos principais conceitos segundo os autores consultados. Na seção seguinte, serão apresentadas propostas de desenvolvimento de OA com o uso das ferramentas do Design Thinking e uma breve discussão sobre o assunto. Em seguida, teremos as considerações finais com as conclusões do estudo e sugestões para pesquisas futuras.

\section{Metodologia}

A presente pesquisa, do ponto de vista dos seus objetivos, pode ser considerada como exploratória porque visa uma maior proximidade com o problema relacionado ao desenvolvimento e prototipação de objetos de aprendizagem. A pesquisa também é interdisciplinar, pois envolve aspectos tanto da educação quanto da computação e do design. Com relação aos procedimentos envolvidos ela é bibliográfica, pois permite que seja possível identificar o conhecimento que já foi produzido acerca do tema que se pretende investigar (SANTOS, KIENEN, CASTIÑEIRA, 2015). Em relação a abordagem, podemos considerála como qualitativa, pois explora aspectos relacionados aos argumentos de diversos especialistas na área estudada, para assim, construir uma interpretação sobre o tema. Segundo Creswell (2010, p. 206), “[...] os procedimentos qualitativos se baseiam em dados de texto e imagem, têm passos únicos na análise de dados e usam estratégias diversas de investigação [...]".

A pesquisa foi dividida em duas fases distintas. Na primeira fase, foi realizada uma busca nas bases de dados SciElo, IEEE Xplore, Web of Science, através da plataforma OUCI e Scopus. Nesta fase foram considerados somente artigos publicados entre o período de 2015 a 2020, evitando assim erros devido o levantamento de dados relativos a conceitos tecnológicos e processos depreciados devido a constante evolução tecnológica. Somente foram selecionados artigos de acesso público ou com acesso e autenticação da rede interna da Universidade Federal de Santa Catarina (UFSC) via VPN ${ }^{5}$, que fizeram referência ao tema de pesquisa nos títulos, resumos e palavras-chave. Também foram selecionados artigos nos idiomas português, inglês e espanhol. Na segunda fase, foram incluídos trabalhos considerados importantes para a compreensão geral do problema. Para a consulta nas plataformas SciElo, IEEE Xplore, Scopus e OUCI foram utilizados os seguintes critérios de busca: (learning object) AND (design thinking) para pesquisas em todos os metadados. Foram consultadas também revistas especializadas com os indicadores Qualis A1, A2 e B1, através da plataforma SciElo.

\footnotetext{
${ }^{5}$ Virtual Private Network
} 


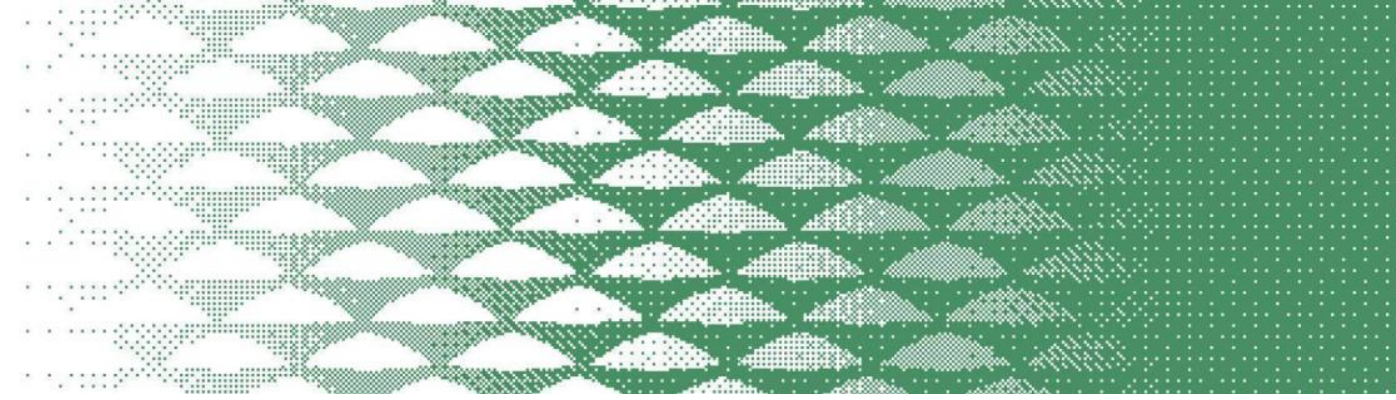

Para a consulta nestas revistas na SciElo foi utilizado o seguinte critério de busca: (learning object) OR (design thinking). Os resultados da pesquisa foram importados para arquivos CSV e posteriormente foram tabulados e organizados em software de planilha eletrônica.

\section{Fundamentação Teórica}

A pesquisa foi dividida em duas fases distintas. Na primeira fase, foi realizada uma consulta nas bases de dados SciElo, IEEE Xplore, WoS e Scopus que retornaram 3153 trabalhos que, após uma primeira análise, foram exportados e organizados em planilhas. Aplicou-se novo filtro sobre títulos, resumos e palavras-chave, resultando em 79 artigos que abordavam o tema de pesquisa. Em uma análise mais minuciosa, considerando a leitura de títulos, resumos e palavras-chave, foram excluídos 8 artigos de acordo com os critérios de inclusão e exclusão, mais especificamente devido a não possuírem acesso público, estarem repetidos ou não se referirem ao tema de pesquisa, resultando em 71 artigos selecionados. Na segunda fase da pesquisa, foi feita a inclusão de outros trabalhos considerados relevantes ao estudo, em um total de 9 artigos e livros. Estes trabalhos então foram organizados em uma planilha, resultando em 80 trabalhos selecionados. Destes, somente 25 foram selecionados para uma leitura na íntegra ou de capítulos específicos relacionados ao estudo com o objetivo de construir uma matriz de síntese. Nas duas fases da pesquisa foi realizada uma análise exploratória dos dados.

\subsection{Objetos de Aprendizagem}

Dentre as tecnologias digitais voltadas para a educação à distância, temos como um dos principais recursos os objetos de aprendizagem, que oferecem muitas possibilidades pedagógicas para ampliação do conhecimento no contexto educacional e que podem ser reutilizados em diversos contextos de ensinoaprendizagem, funcionando assim como facilitadores deste processo (GROSSI, LEAL, 2020). Esta capacidade que objetos de aprendizagem possuem de serem reaproveitados remetem ao conceito de orientação à objetos, onde o código fonte pode e deve ser reutilizado em diversos contextos de desenvolvimento de sistemas (ULBRICHT, VILLAROUCO, FADEL, 2017). Estes recursos podem então ser armazenados em bibliotecas online e repositórios digitais, que poderão ser acessados de modo síncrono ou assíncrono pelos alunos à distância (GOODSETT, 2020, tradução nossa). Outra característica importante dos objetos de aprendizagem é a necessidade de haver uma explicitação de seus objetivos pedagógicos (GROSSI, LEAL, 2020). Assim, não se pode confundir um objeto de aprendizagem com um conteúdo digital, não somente pelos seus aspectos de orientação à objetos, mas também por possuir um aspecto pedagógico incluso (BORBA et al., 2016, tradução nossa).

Um objeto de aprendizagem também pode ser considerado como um caminho percorrido pelo aluno, com um ponto de partida e outro ponto de chegada, no qual deve ocorrer a aprendizagem (CANTO FILHO, LIMA, TAROUCO, 2017), ou ainda, "uma categoria de aprendizagem multimídia auxiliar" (MASCHIO, CORREIA, 2020, tradução nossa, p. 202). Objetos de aprendizagem também devem conter aspectos técnicos, como o uso de metadados, com o fim de serem facilmente indexados, recuperados e reutilizados em repositórios digitais (VICARI et al., 2010). Estes metadados podem ser compreendidos como dados sobre dados. De acordo com Pottker, Ferneda e Moreiro-González (2018), os metadados são recomendações de Web Semântica que descrevem características de recursos informacionais. Estes metadados são transformados em seus formatos nativos em $\mathrm{RDF}^{6}$, um formato recomendado para apresentação de dados em Web Semântica, de acordo com o Linked Data e disponibilizados por meio de URIs $^{7}$ (JEVSIKOVA, BERNIUKEVICIUS, KURILOVAS, 2017, tradução nossa). O RDF e o Linked Data (Dados Vinculados), são usados para anotar e classificar recursos de acordo com a similaridade entre outros critérios na construção de categorias de recursos (JEVSIKOVA,

\footnotetext{
${ }^{6}$ Resource Description Framework

${ }^{7}$ Uniform Resource Identifiers
} 


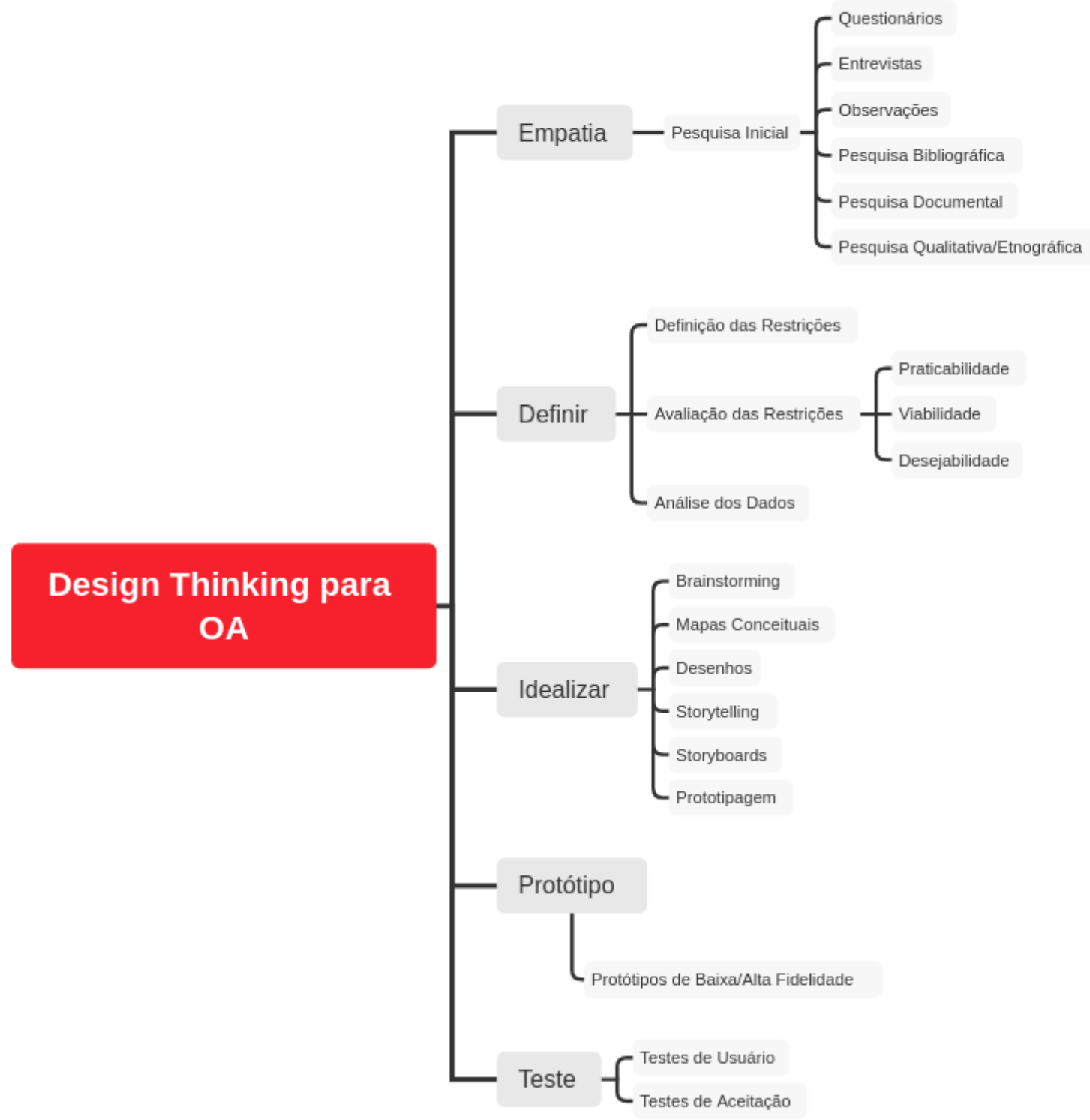

Imagem 1 - Mapa mental de Design Thinking para OA.

Fonte: Autoria própria (2021)

A partir destas sugestões de abordagem dos processos de produção de objetos de aprendizagem com aplicação de ferramentas de Design Thinking, poderemos obter uma maior qualidade e satisfação por parte dos usuários no uso destes recursos. No entanto, o processo de desenvolvimento de OA é formado por quatro etapas: desenvolvimento do conteúdo - que envolve o design instrucional não abordado nesta pesquisa e a aplicação do Design Thinking no projeto de OA -, empacotamento, distribuição e armazenamento em repositórios digitais que também não foram mencionados com mais detalhes neste trabalho (BORBA et al., 2016, tradução nossa).

\section{Considerações Finais}

Neste estudo, foi demonstrado a importância da inclusão de ferramentas de Design Thinking em um processo de desenvolvimento de objetos de aprendizagem, de maneira a aumentar a qualidade e 
CANTO FILHO, Alberto Bastos dos; LIMA, José Valdeni de; TAROUCO, Liane Margarida Rockenbach. Mapas Conceituais de Projeto: uma ferramenta para projetar objetos de aprendizagem significativa. in: Ciência \& Educação, Ciênc. educ. Bauru: 2017.

CARPENEDO, Daiana Freitas; MONBACH, Jaline Gonçalves; COUTINHO, Renato Xavier; RAVASIO, Marcele Teixeira Homrich; RODRIGUES, Ricardo Antonio; ROSSI, Fábio Diniz. Experience in creating learning objects in a professional master's degree in education. in: International Journal of Information and Education Technology, International Journal of Information and Education Technology. Singapore: 2020.

CRESWELL, John W. Projeto de Pesquisa: Métodos Qualitativo, Quantitativo e Misto, $3^{\circ}$ ed. Artmed. Porto Alegre: 2010.

ELLWANGER, Cristiane. Design de Interação, Design Experiencial e Design Thinking: A tríade que permeia o escopo de desenvolvimento de sistemas computacionais interativos. in: Nuevas Ideas en Informática Educativa TISE 2013, TISE. Porto Alegre: 2013.

FERRO, Gláucia de Salles. Design thinking: modelo aplicável em desafios complexos e em inovação, Pimenta Cultural. São Paulo: 2020.

GOODSETT, Mandi. Assessing the potential for critical thinking instruction in information literacy online learning objects using best practices. in: Communications in Information Literacy, Communications in Information Literacy. Portland: 2020.

GROSSI, Márcia Gorett Ribeiro; LEAL, Débora Cristina Cordeiro Campos. Análise dos Objetos de Aprendizagem Utilizados em Curso Técnico de Meio Ambiente a Distância. in: Ciência \& Educação, Ciênc. educ. Bauru: 2020.

HSIAO, Hsien-Sheng; YU, Kuang-Chao; CHANG, Yu-Shan; CHIEN, Yu-Hung; LIN, Kuen-Yu; LIN, ChienYu; CHEN, Jyun-Chen, CHEN, Jheng-Han; LIN, Yi-Wei. The Study on Integrating the Design Thinking Model, in: 7th World Engineering Education Forum (WEEF) and STEM Activity Unit for Senior High School Living Technology Course, IEEE. Kuala Lumpur: 2017.

JEVSIKOVA, Tatjana; BERNIUKEVICIUS, Andrius; KURILOVAS, Eugenijus. Application of resource description framework to personalise learning: Systematic review and methodology. in: Informatics in Education, Institute of Mathematics and Informatics. Vilnius: 2017.

LEE, C.; WONG, D. An entrepreneurial narrative media-model framework to knowledge building and open co-design for smart cities. in: Computing Conference, IEEE. Londres: 2017.

LEVY, Meira. Promoting the Elicitation of Usability and Accessibility Requirements in Design Thinking: Using a Designed Object as a Boundary Object. in: IEEE 25th International Requirements Engineering Conference Workshops (REW), IEEE. Lisboa: 2017.

LIU, Yang; WEI, Xiaodong; LI, Zhe. Improving Design and Creative Ability of High School Students through Project Based Learning. in: International Joint Conference on Information, Media and Engineering (IJCIME), IEEE. Osaka: 2019. 
MALDONADO, Jorge J.; BERMEO, Jorge L.; PACHECO, Guillermo. Assessing a Methodological Proposal for the Design, Creation and Evaluation of Learning Objects Oriented to Educators with Diverse Educational and Technological Competencies. in: CLEI Electronic Journal, CLEIej. Montevideo: 2016.

MASCHIO, Alexandre V.; CORREIA, Nuno M. R. Digital learning object for audiovisual production. in: International Journal of Information and Education Technology, International Journal of Information and Education Technology. Singapore: 2020.

POTTKER, Luciana Maria Vieira; FERNEDA, Edberto; MOREIRO-GONZÁLEZ, José Antonio. Mapeamento relacional entre padrões de metadados educacionais. in: Perspectivas em Ciência da Informação, Perspect. ciênc. inf. Belo Horizonte: 2018.

QUEIROS, Leandro Marques; SILVEIRA, Denis Silva da; CORREIA-NETO, Jorge da Silva; VILAR, Guilherme. LODPRO: learning objects development process. in: Journal of the Brazilian Computer Society, Springer London. London: 2016.

RAJASHEKHARAIAH, k. m. m.; PAWAR, Manjula; PATIL, S. Mahesh; KULENAVAR, Nagaratna; JOSHI, G. H. Design Thinking Framework to Enhance Object Oriented Design and Problem Analysis Skill in Java Programming Laboratory: An Experience. in: IEEE 4th International Conference on MOOCs, Innovation and Technology in Education (MITE), IEEE. Madurai: 2016.

SANTOS, Pedro António dos; KIENEN, Nádia; CASTIÑEIRA, Maria Inés. Metodologia da Pesquisa Social: Da Proposição de um Problema à redação e Apresentação do Relatório, Ed. Atlas. Barueri: 2015.

SUSMITHA, V. ; AKSHAY, N. ; VENNILA, V. ; MURALEEDHARAN, A. ; NAIR, R. ; VELAYUDHAN, A. ; ALKOYAK, M. ; BHAVANI, R. R. All Aboard for the Joy of Making! Teaching User Centered Design and Tinkering to Middle School Children in India. in: 2018 IEEE Tenth International Conference on Technology for Education (T4E), IEEE. Chennai: 2018.

TODOS PELA EDUCAÇÃO. Anuário Brasileiro da Educação Básica, Ed. Moderna, São Paulo: 2020.

ULBRICHT, Vania Ribas; VILLAROUCO, Vilma; FADEL, Luciane. Protótipos Funcionais de Objetos de Aprendizagem, Pimenta Cultural. São Paulo: 2017.

VICARI, Rosa Maria; BEZ, Marta; SILVA, Júlia Marques Carvalho da; RIBEIRO, Alexandre; GLUZ; João Carlos; PASSERINO, Liliana; SANTOS, Elder; PRIMO, Tiago; ROSSI, Luiz; BORDIGNON, Alexandro; BEHAR, Patricia; RAYMUNDO FILHO; ROESLER, Valter. Proposta Brasileira de Metadados para Objetos de Aprendizagem Baseados em Agentes (OBAA). in: Cinted-ufrgs Novas Tecnologias na Educação, Cinted-ufrgs. Porto Alegre: 2010. 